\title{
Concentration of proinflammatory cytokines in patients with ulcers as a complication of type 2 diabetes mellitus
}

\author{
Daria Kupczyk ${ }^{1}$, Rafał Bilski¹, Andrzej Przewięźlikowski², Renata Studzińska³, Alina Woźniak ${ }^{1}$
}

'Department of Medical Biology and Biochemistry, Ludwik Rydygier Collegium Medicum in Bydgoszcz, Nicolaus Copernicus University in Torun, Poland

2Private Nursing Practice Andrzej Przewięźlikowski, Torun, Poland

${ }^{3}$ Department of Organic Chemistry, Ludwik Rydygier Collegium Medicum in Bydgoszcz, Nicolaus Copernicus University in Torun, Poland Adv Dermatol Allergol 2021; XXXVIII (5): 767-772

DOI: https://doi.org/10.5114/ada.2020.96702

\begin{abstract}
Introduction: Difficult healing of chronic wounds is a serious problem for modern medicine. It leads to ulceration, especially in conditions such as diabetic foot syndrome or chronic venous insufficiency. This may be a result of chemical, physical, thermal or biological factors, among others. Analysis of mediators and molecular factors released by the abovementioned structure helps to better understand the mechanism of healing of chronic wounds and the formation of ulcers.

Aim: To assess excretion of selected cytokines in patients with ulcerations as a complication of diabetes mellitus type 2.

Material and methods: Seventeen patients aged 68-87 took part in the assessment of wound healing in patients with ulceration in the course of diabetes mellitus type 2 . The control group consisted of 21 healthy patients aged 32-62. In the blood serum bFGF, TNF- $\alpha$, IL-4, TGF- $\beta 1$, TGF- $\beta 2$ and TGF- $\beta 3$ were determined.

Results: A significant difference was found in bFGF, IL-4, TGF- $\beta 1$, TGF- $\beta 2$, and TGF- $\beta 3$ levels. Concentration of bFGF was $12 \%$ lower in patients with ulcers than in the non-ulcerated control group $(p=0.013)$. IL-4 concentration was $46 \%$ lower in patients with ulcers than in the non-ulcerated control group $(p=0.002)$. TGF- $\beta 1$, TGF- $\beta 2$ and TGF- $\beta 3$ concentrations were also lower in the group of patients with ulcers compared to those in the non-ulcerated control group.

Conclusions: Reduced concentrations of selected cytokines and growth factors may indicate abnormal activity of the cells that secrete them and affect the healing process of chronic wounds, hindering and delaying the healing process.
\end{abstract}

Key words: type 2 diabetes, chronic venous insufficiency, ulcers, cytokines, growth factors.

\section{Introduction}

Diabetes is a chronic metabolic disease. It is characterized by elevated blood glucose levels and the occurrence of complications within large and small vessels of the nature of micro- and macroangiopathy [1]. It is now considered a global epidemic [2]. In type 2 diabetes, insulin is produced by pancreatic $\beta$ cells, but the body's target tissues are less sensitive to this hormone [3-5]. In diabetic patients, chronic hyperglycemia contributes to the development of pro- and antioxidant imbalance $[6,7]$. One of the most serious complications is the diabetic foot syndrome, as it can lead to severe disability in a short time [8-11]. Diabetic neuropathy and ischemia of the lower extremities are the most important etiologi- cal factors of foot ulcers. In turn, as a result of atherosclerotic changes that arise in the vessels of the lower extremities, ischemia occurs, causing a deterioration of the healing process, fighting infection or hindering the penetration of the antibiotic to the site of infection [2]. Healing disorders, T cell dysfunction and immune disorders are factors that increase the risk of limb amputation in patients with foot ulcer diabetes $[12,13]$. Hence, it seems important to understand the molecular mechanisms associated with the healing of chronic wounds and factors hindering this process $[14,15]$. The most common venous diseases include lower limb varicose veins, chronic venous insufficiency (CVI), venous leg ulcers, as well as thrombophlebitis or deep vein thrombosis [16]. There are many factors that predispose to their occurrence.

Address for correspondence: Daria Kupczyk PhD, Department of Medical Biology and Biochemistry, Ludwik Rydygier Collegium Medicum, Nicolaus Copernicus University, Bydgoszcz, Poland, e-mail: dariak@cm.umk.pl Received: 20.03.2020, accepted: 23.04.2020. 
The main factor leading to the development of venous system diseases is venous hypertension, caused by venous obstruction or narrowing, impaired venous valve function or pressure on the veins [17]. As a result of blood stasis, leukocytes move outside the vessel wall and stimulate it towards the development of the inflammatory process. It also results in the activation of proteolytic enzymes or free radicals. As a result of the venous load and the development of the inflammatory reaction, capillary permeability increases and edema develops. A troublesome and difficult to cure consequence of venous system diseases is venous leg ulcers. These are secondary lesions, in which necrotic tissue breaks down due to insufficient blood supply. Pathological changes include the epidermis, dermis and subcutaneous tissue [18].

\section{Aim}

The aim of this study is to assess the concentration of selected proinflammatory cytokines and growth factors in patients with ulceration in the course of diabetes mellitus type 2. The obtained results will make it easier to understand the role of individual cells, especially their secreted cytokines and growth factors during wound healing, which will allow the introduction of more effective methods of treatment.

\section{Material and methods}

The study included a group of 17 patients, including 10 women and 7 men, aged 68-87. The average age was $75 \pm 6$ years. The study group consisted of patients from a specialist surgery whose activities include surgical nursing and chronic wound treatment. The criteria for including patients in the study were as follows: informed consent, diagnosed type 2 diabetes, and the presence of its complications in the form of ulcers. The exclusion criteria were: no consent to participate in the study, cancer, inflammatory diseases of the osteoarticular system, heart and circulatory system, dementia, Alzheimer's and Parkinson's diseases. Patients with ulceration around the foot and lower leg in the course of the complications of type 2 diabetes mellitus were qualified for the study. Patients who came to the office, were under the specialist care of medical staff due to the fact that the process of chronic wound treatment is long and requires taking a number of actions, such as wound development, the use of total contact cast or vacuum dressings/negative pressure wound therapy or the use of devices enabling fast and accurate transdermal measurement of oxygen partial pressure or measurement of systolic blood pressure on the fingers of the lower and upper limbs.

The control group consisted of 21 patients aged 32-62 (12 women and 9 men; average age $45 \pm 9$ years). The control group was selected from patients without chronic diseases, without signs of inflammation and neurodegenerative processes that are characteristic of old age.

The material for biochemical tests was venous blood collected in the amount of approx. $5 \mathrm{ml}$ from the ulnar vein into tubes devoid of anticoagulant. Blood was collected in the morning. Then, the collected material was transported to the Department of Medical Biology and Biochemistry of the Collegium Medicum in Bydgoszcz at the Nicolaus Copernicus University in Torun. The study was permitted by the bioethics committee of Nicolaus Copernicus University in Torun (permission number KB 344/2018). The procedures followed the Helsinki Declaration of 1975 , as revised in 2000. Serum was obtained from blood collected in tubes without anticoagulant, by centrifuging the material for $5 \mathrm{~min}$ at $5000 \times \mathrm{g}$, and then transferred to Eppendorf tubes and frozen at $-80^{\circ} \mathrm{C}$. The serum prepared in this way was stored for the determination of tumor necrosis factor $\alpha$ (TNF- $\alpha$ ), interleukin (IL)-4, basic fibroblast growth factor (bFGF) and three isoforms transforming growth factor $\beta$ (TGF- $\beta$, i.e. TGF- $\beta 1$, TGF- $\beta 2$, TGF- $\beta 3$ ). The analysis of selected cytokines was performed using the multi ELISA method using the Bio-Plex Pro Human Cytokine Grp I Panel from BIO-RAD cat \# M500KCAFOY. The limit of detection for IL-4 was $0.09 \mathrm{pg} / \mathrm{ml}$, for TNF- $\alpha$ - $1.13 \mathrm{pg} / \mathrm{ml}$, for $\mathrm{bFGF}-2.54 \mathrm{pg} / \mathrm{ml}$, for TGF- $\beta 1-3.9 \mathrm{pg} /$ $\mathrm{ml}$, for TGF- $\beta 2-1.9 \mathrm{pg} / \mathrm{ml}$, and for TGF- $\beta 3-0.5 \mathrm{pg} / \mathrm{ml}$. The assay precision using coefficient of variation, intraand inter-assay was the following, for IL-4 -3.2\% and 1.9\%, for TNF- $\alpha-3.5 \%$ and $3.0 \%$, for bFGF $-3.1 \%$ and $2.4 \%$, for TGF- $\beta 1-4.5 \%$ and $4.9 \%$, for TGF- $\beta 2-6.3 \%$ and $9.1 \%$, and for TGF- $\beta 3-6.9 \%$ and $8.2 \%$.

\section{Statistical analysis}

Statistical analysis of biochemical parameters was carried out using the Statistica 13.3 program. The distribution normality was first tested using the Shapiro-Wilk test. The tested parameters met the criteria of normal distribution. The $t$ test for independent variables was used for further analysis. The results with a $p$-value lower than 0.05 were considered as statistically significant. The correlations between pairs of parameters were calculated using the Spearman method.

\section{Results}

A total of 38 patients participated in the study. The study group consisted of 17 patients aged between 68 and 87 years old. The group included 10 women and 7 men. The control group consisted of 21 patients including 12 women and 9 men aged between 32 and 62 years old. Neither group taking part in the study had co-morbidities.

\section{bFGF concentration}

The average concentration of bFGF in the serum of subjects in the study group was $12 \%$ lower than in the 
Table 1. Presentation of average concentration values of selected biochemical parameters in the study and control groups

\begin{tabular}{lccccc}
\hline Parameter & \multicolumn{2}{c}{ Study group, $n=17$} & \multicolumn{2}{c}{ Control group, $n=21$} & \multirow{2}{*}{$P$-value } \\
\cline { 2 - 5 } & Mean & Standard deviation & Mean & Standard deviation & \\
\hline bFGF $[\mathrm{pg} / \mathrm{ml}]$ & 40.95 & 6.11 & 46.35 & 6.44 & 0.013 \\
\hline TNF- $\alpha[\mathrm{pg} / \mathrm{ml}]$ & 49.74 & 34.90 & 46.17 & 9.95 & 0.656 \\
\hline IL-4 $[\mathrm{pg} / \mathrm{ml}]$ & 2.47 & 0.83 & 4.60 & 2.19 & 0.002 \\
\hline TGF- $\beta 1[\mathrm{pg} / \mathrm{ml}]$ & 43053.98 & 20155.37 & 70574.67 & 36105.23 & 0.005 \\
\hline TGF- $\beta 2[\mathrm{pg} / \mathrm{ml}]$ & 569.00 & 286.90 & 730.70 & 164.48 & 0.035 \\
\hline TGF- $\beta 3[\mathrm{pg} / \mathrm{ml}]$ & 111.92 & 55.03 & 155.69 & 51.90 & 0.012 \\
\hline
\end{tabular}

control group. The level of statistical significance was $p=0.013$.

\section{TNF- $\alpha$ concentration}

A statistically significant difference in TNF- $\alpha$ concentration between the study and control group was not observed $(p=0.656)$ (Table 1$)$.

\section{IL-4 concentration}

A statistically significant difference in IL-4 concentration between the groups was found. IL-4 concentration in the study group was $46 \%$ lower than in the control group $(p=0.002)$.

\section{TGF- $\beta 1$ concentration}

The concentration of TGF- $\beta 1$ was $39 \%$ lower in the study group compared to the control group $(p=0.005)$.

\section{TGF- $\beta 2$ concentration}

$22 \%$ lower TGF- $\beta 2$ concentration was observed in the study group compared to the control group $(p=0.035)$.

\section{TGF- $\beta 3$ concentration}

A statistically significant difference was found in the levels of transforming $\beta$-growth factor isoform 3 between individual groups. Similarly, the concentration of TGF- $\beta 3$ in the study group was $28 \%$ lower than in the control group $(p=0.012)$ (Table 1$)$.

\section{Correlation analysis in study and control groups}

There were positive correlations between parameters in both groups. The detailed results are shown in Table 2.

Statistically significant positive correlations between TGF- $\beta 1$ and TGF- $\beta 3$ ( $r=0.77, p<0.01)$, TGF- $\beta 1$ and TGF- $\beta 2$ $(r=0.82, p<0.01)$, TGF- $\beta 2$ and TGF- $\beta 3(r=0.91, p<0.01)$, TGF- $\beta 1$ and bFGF ( $r=0.70, p<0.01)$, TGF- $\beta 1$ and TNF- $\alpha$ $(r=0.69, p<0.01)$, TGF- $\beta 2$ and bFGF $(r=0.50, p=0.04)$ (Figure 1) were observed in the study group (Table 2 ).

Statistically significant positive correlations between TGF- $\beta 1$ and TGF- $\beta 3$ (0.86, $p<0.01)$, TGF- $\beta 1$ and TGF- $\beta 2$ (0.74,
Table 2. Statistically significant correlation coefficients between the parameters measured in patients with ulcers and in non-ulcer control group

\begin{tabular}{lccc}
\hline Group & Parameter & $r$ & $P$-value \\
\hline Study group & TGF- $\beta 2 /$ TGF- $\beta 3$ & 0.91 & $<0.01$ \\
\cline { 2 - 4 } & TGF- $\beta 1 /$ TGF- $\beta 3$ & 0.77 & $<0.01$ \\
\cline { 2 - 4 } & TGF- $\beta 1 /$ TGF- $\beta 2$ & 0.82 & $<0.01$ \\
\cline { 2 - 4 } & bFGF/TGF- $\beta 2$ & 0.50 & 0.04 \\
\cline { 2 - 4 } & bFGF/TGF- $\beta 1$ & 0.70 & $<0.01$ \\
\cline { 2 - 4 } Control group & TNF- $\alpha /$ TGF- $\beta 1$ & 0.69 & $<0.01$ \\
\cline { 2 - 4 } & TGF- $\beta 2 /$ TGF- $\beta 3$ & 0.77 & $<0.01$ \\
\cline { 2 - 4 } & TGF- $\beta 1 /$ TGF- $\beta 3$ & 0.86 & $<0.01$ \\
\cline { 2 - 4 } & TGF- $\beta 1 /$ TGF- $\beta 2$ & 0.74 & $<0.01$ \\
\cline { 2 - 4 } & IL-4/TNF- $\alpha$ & 0.47 & 0.04 \\
\cline { 2 - 4 } & bFGF/TNF- $\alpha$ & 0.51 & 0.02 \\
\hline
\end{tabular}

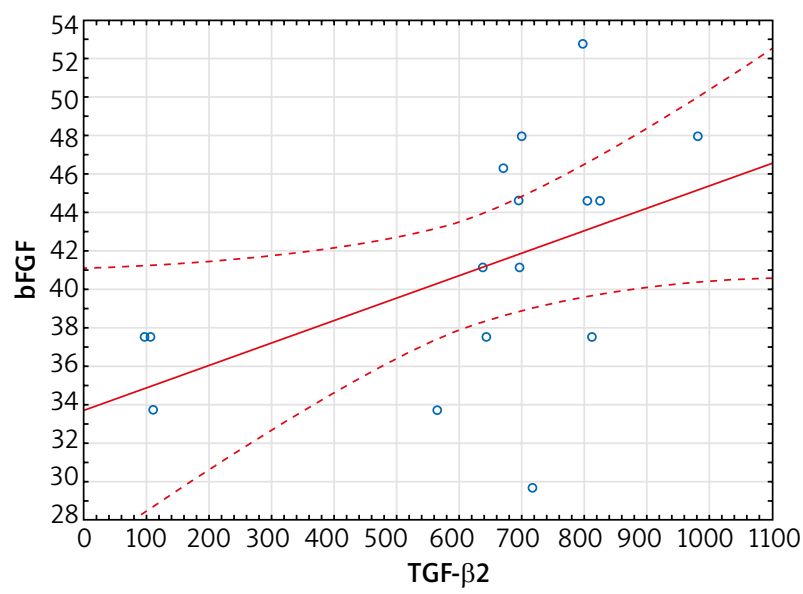

Figure 1. Linear regression of bFGF concentration versus TGF- $\beta 2$ concentration in the serum of subjects in the study group $(r=0.50, p=0.04)$

$p<0.01)$, TGF- $\beta 2$ and TGF- $\beta 3$ ( $r=0.77, p<0.01), \mathrm{IL}-4$ and TNF- $\alpha(r=0.47, p=0.04)$ (Figure 2), bFGF and TNF- $\alpha(r=$ $0.51, p=0.02$ ) were observed in the control group (Table 2 ). 


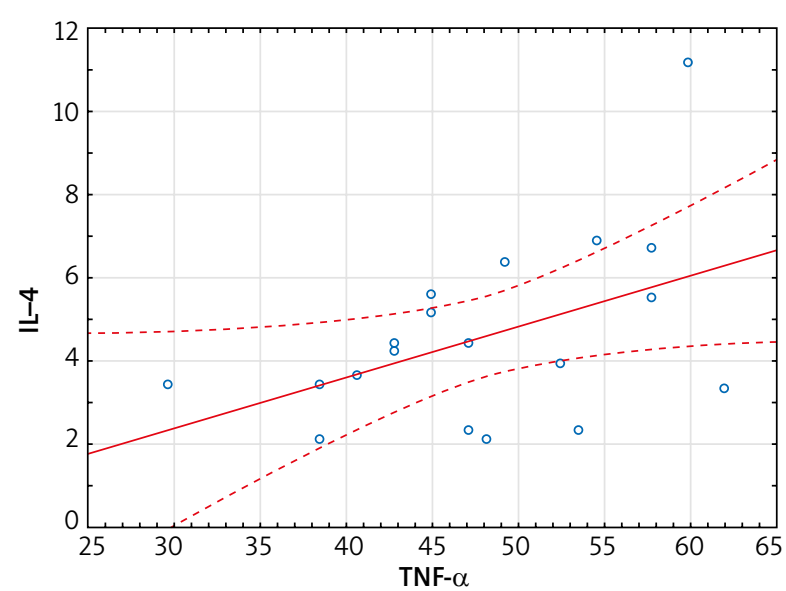

Figure 2. Linear regression of IL-4 concentration versus TNF- $\alpha$ concentration in the serum of subjects in the control group $(r=0.47, p=0.04)$

\section{Discussion}

Our study showed statistically significant changes in the levels of growth factors (bFGF, TGF- $\beta 1$, TGF- $\beta 2$, TGF- $\beta 3$ ) as well as IL-4 between the study and control group. Statistically significant correlations between growth factors in the study group were observed. Correlation analysis within the control group showed positive correlations between growth factors, as well as between TNF- $\alpha$ and IL-4, and TNF- $\alpha$ and bFGF.

In patients with type 2 diabetes the quality of life deteriorates as complications, such as ulcerations, develop. Pro-inflammatory cytokines, involved in the pathogenesis of this disease, can accelerate the development of complications associated with it.

The mechanism of action of modern dressings helps in cleaning the wound of necrotic tissues and supports granulation processes. Unfortunately, despite the use of even the best techniques, chronic and extensive venous ulcers are difficult and long-healing lesions [18]. In the wound healing process, there are successive phases of inflammation, namely, proliferation and remodeling. After tissue damage, the blood vessels and platelets are exposed to extracellular matrix proteins, which leads to the formation of a clot. This phenomenon is accompanied by the secretion of, among other secretions, TNF- $\alpha$ with simultaneous influx of neutrophils, monocytes and mast cells. This is to stimulate angiogenesis, eliminate microorganisms or regenerate tissues. It also enables cell migration, and the resulting clot also becomes a source of wound healing mediators [19]. Tumor necrosis factor is an example of a multifunctional pro-inflammatory cytokine involved in the regulation of the immune response, hematopoiesis and inflammation. It is a polypeptide produced mainly by monocytes, macrophages and lymphocytes, but also, in smaller quantities, by neutrophils, fibroblasts, keratinocytes and mast cells [20]. Ritsu et al. evaluated the wound healing process on mouse models. They then measured the concentration of TNF- $\alpha$ in homogenate supernatants collected from wounds at various intervals. They detected the presence of this cytokine shortly after the wound was formed and its level increased within the first few hours, reaching a maximum concentration on the first day, and then decreased [21]. In turn, Frank et al. studied the effect of this cytokine on epithelization of the wound and in vivo neovascularization in studies conducted on mice. They found that TNF- $\alpha$ accelerates these processes and compensates for the negative impact of macrophage reduction, which can have a direct impact on wound healing [22]. Our study did not show a statistically significant difference in the TNF- $\alpha$ concentration between the test group and the control group. This may be due to the fact that TNF- $\alpha$ may reach high concentrations, but just after injury. Activated platelets play a key role in clot formation during hemostasis after aggregation and adhesion to exposed collagen surfaces. This process is mediated by many factors released by platelets, including transforming growth factor $\beta$. In turn, macrophage activation can be a classic M1 phenotype (highly pro-inflammatory) or alternatively M2 phenotype. The alternative type involves IL-4 and/ or IL-13, which release growth factors derived from macrophages. These include bFGF and TGF $\beta$. This suggests an important role for alternative macrophage activation in the healing phase [23]. Research by Salmon-Ehr et al. shows that interleukin 4 may also be involved in wound healing. Expression of this cytokine was detected in mice on the first day after injury and was still high until the fourth day and then gradually decreased. Local application of IL-4 significantly accelerated the rate of wound healing [24]. In our study, the concentration of IL-4 in the group of subjects was reduced compared to the control group. According to the research of Salmon-Ehr et al. and based on our own research, it can be concluded that the reduced values of the tested cytokine in patients with ulcers may adversely affect the wound healing process, and its local administration may contribute to an increase in the rate of chronic wound healing. The activity of cells that are involved in wound healing is strictly regulated by the microenvironment, which is formed, among others, by fibroblast growth factor (FGF) or transforming growth factor (TGF). FGF factors, which include 22 polypeptides, are active mitogens that stimulate cell proliferation of ecto-, meso- and endodermal origin [25]. They also induce the migration and proper differentiation of cells and fulfill a protective role in the state of cellular stress [26]. A particularly important role is attributed to the bFGF factor, which is produced immediately after wounding by damaged endothelial cells of blood vessels, and in subsequent stages of the healing process by incoming macrophages to the wound [27]. Choi et al. used structurally stabilized bFGF in dressings in mice to improve wound healing in type 1 and type 2 diabetes [28]. 
Lv et al., in a study performed on rabbits in which incisions were made to produce infectious wounds, observed that bFGF expression promotes the healing of infectious wounds [29]. In the group of patients we examined, we observed a statistically significant difference in the concentration of this factor between the test group and the control group. bFGF concentration was lower in the group of patients with ulcers, which confirms the effect of this parameter on the healing process. Lowering the concentration of bFGF contributes to the healing process in the group of patients studied. Similarly, in the group of patients with ulcers we studied, the concentrations of the three TGF- $\beta 1-3$ proteins were statistically significantly lower in the study group compared with the control group. In contrast, Gazaerly et al. assessed the effect of TGF- $\beta 1$ on wound healing as a complication of diabetes in rats. The team of researchers observed in diabetic rats treated with TGF- $\beta 1$ a significant improvement in healing compared to diabetic rats [30]. In turn, Wang et al. applied the wound antimicrobial agent povidone-iodine (PVI) in rats to wounds, which, by increasing TGF- $\beta$ expression, led to increased production of granulation tissue [31]. TGF- $\beta$ is primarily produced by platelets present in the wound area just after tissue damage. It works chemotactically on fibroblasts, neutrophils and macrophages [32]. It is these cells in the later stages of healing that produce, in addition to cytokines and growth factors, also TGF- $\beta$. Cytokines and growth factors such as FGF or TGF- $\beta$, which are released during the inflammatory phase, stimulate the formation of fibrous tissue, and the transition from the inflammatory to proliferative phase depends mainly on the activity of macrophages [33]. In turn, during the proliferation phase, granulation tissue is formed, which is formed by a loose network of collagen fibers, hyaluronic acid or fibronectin. Their production depends, among other factors, on the presence of TGF- $\beta 1$ or TGF- $\beta 2$ [34]. In our study, a statistically significant correlation between bFGF and TGF- $\beta 2$ was found in the study group, while there was no statistically significant correlation between those parameters in the control group. Both of those parameters participate in the wound healing process through production of monocytes, macrophages, and endothelial cells; therefore, the correlated decrease of those parameters may be related to the impaired wound healing process and the creation of chronic wounds. Those results agree with Uchi et al. and Jude et al. [35, 36]. In our study there was a statistically significant correlation between IL-4 and TNF- $\alpha$ in the control group, while there was no significant correlation between those parameters in the study group. There is a weaker inflammatory response in the study group; therefore, IL- 4 and TNF- $\alpha$ concentrations do not correlate within patients with ulcers. Inflammatory cytokines have a large impact on the wound healing process in patients with ulcers $[23,24,37]$. Current reports indicate that extracellular vesicles could induce macrophages to form anti-inflammatory $\mathrm{M} 2$ phenotypes; therefore, they may affect the regulation of metabolism and insulin resistance [38-40].

The main limitation of our study was gathering a group of patients with ulcers and without co-morbidities. The promising results presented in our paper may be the basis for continuation in order to collect and compile data for a larger group of patients and applying for more funds allocated for this purpose.

\section{Conclusions}

The results of our research on a group of patients with ulcerations in the course of diabetic foot syndrome and chronic venous insufficiency show that the abnormal activity of cells and their secreted cytokines or growth factors affect the healing process of chronic wounds, hindering and delaying the healing process. Therefore, from a clinical point of view, it seems that knowledge about the mechanisms of healing is extremely important, and understanding the role of individual cells, cytokines or growth factors during the wound healing process will allow the introduction of more effective methods of treatment.

\section{Conflict of interest}

The authors declare no conflict of interest.

\section{References}

1. Genuth S, Eastman R, Kahn R, et al. Implications of the United Kingdom Prospective Diabetes Study. Diabetes Care 2003; 1: 27-31.

2. Lavery LA, Oz OK, Bhavan K, et al. Diabetic foot syndrome in the twenty-first century. Clin Podiatr Med Surg 2019; 36: 355-9.

3. Abdul-Ghani MA, Tripathy D, DeFronzo RA. Contributions of beta-cell dysfunction and insulin resistance to the pathogenesis of impaired glucose tolerance and impaired fasting glucose. Diabetes Care 2006; 29: 1130-9.

4. Del Prato S. Role of glucotoxicity and lipotoxicity in the pathophysiology of Type 2 diabetes mellitus and emerging treatment strategies. Diabet Med 2009; 26: 1185-92.

5. Wu Y, Ding Y, Tanaka Y, Zhang W. Risk factors contributing to type 2 diabetes and recent advances in the treatment and prevention. Int J Med Sci 2014; 11: 1185-200.

6. Baynes JW, Thorpe SR. Role of oxidative stress in diabetic complications, a new perspective on an old paradigm. Diabetes 1999; 48: 1-9.

7. Reusch JE. Diabetes, microvascular complications, and cardiovascular complications: what is it about glucose? I Clin Invest 2003; 112: 986-8.

8. Singh N, Armstrong DG, Lipsky BA. Preventing foot ulcers in patients with diabetes. JAMA 2005; 293: 217-28.

9. Lavery LA, Ashry HR, van Houtum W, et al. Variation in the incidence and proportion of diabetes related amputations in minorities. Diabetes Care 1996; 19: 48-52. 
10. Lavery LA., van Houtum W, Astry HR, et al. Diabetes related lower extremity amputations disproportionately affect blacks and Mexican Americans. South Med J 1999; 92: 593-9.

11. Pecoraro RE, Reiber GE, Burgess EM. Pathways to diabetic limb amputations: basis for prevention. Diabetes Care 1990; 13: 513-21.

12. Geerlings SE, Hoepelman Al. Immune dysfunction in patients with diabetes mellitus. FEMS Immunol Med Microbiol 1999; 26: 259-65.

13. Blakytny R, Jude $E$. The molecular biology of chronic wounds and delayed healing in diabetes. Diabet Med 2006; 23: 594 608.

14. Han G, Ceilley R. Chronic wound healing: a review of current management and treatments. Adv Ther 2017; 34: 599-610.

15. Eklöf B, Rutheford RB, Bergan JJ, et al. Revision of the CEAP classification for chronic venous disorders: consensus statement. J Vasc Surg 2004; 40: 1248-52.

16. Schmitz M. German S3-Clinical Practice Guideline: Topical treatment of chronic wounds at patients with the risks of occlusive arterial disease, diabetes mellitus, chronic venous insufficiency: importance and consequences for wound management. Wound Med 2013; 1: 37-40.

17. Bergan J. Molecular mechanisms in chronic venous insufficiency. J Dtsch Dermatol Ges 2006; 4: 627-8.

18. Anderson K, Hamm RL. Factors that impair wound healing. J Am Coll Clin Wound Spec 2014; 4: 84-91.

19. Page MJ, Bester J, Pretorius E: The inflammatory effects of TNF- $\alpha$ and complement component 3 on coagulation. Sci Rep 2018; 8: 1812.

20. Dinarello CA. Proinflammatory cytokines. Chest 2000; 118: 503-8.

21. Ritsu M, Kawakami K, Kanno E, et al. Critical role of tumor necrosis factor- $\alpha$ in the early process of wound healing in skin. J Dermatol Dermatol Surg 2017; 21: 14-9.

22. Frank J, Born K, Barker JH, et al. In vivo effect of tumor necrosis factor alpha on wound angiogenesis and epithelialization. Eur J Trauma 2003; 29: 208-19.

23. Qing C. The molecular biology in wound healing \& non-healing wound. Chin J Traumatol 2017; 20: 189-93.

24. Salmon-Ehr V, Ramont L, Godeau G, et al. Implication of interleukin-4 in wound healing. Lab Invest 2000; 80: 1337-43.

25. Gibran NS, Isik FF, Heimbach DM, et al. Basic fibroblast growth factor in the early human burn wound. I Surg Res 1994; 56: 226-34.

26. Itoh N, Ornitz DM. Fibroblast growth factors: from molecular evolution to roles in development, metabolism and disease. J Biochem 2011; 149: 121-30.

27. Fiddes JC, Hebda PA, Hayward P, et al. Preclinical woundhealing studies with recombinant human basic fibroblast growth factor. Ann NY Acad Sci 1991; 638: 316-28.

28. Choi SM, Lee KM, Kim HJ, et al. Effects of structurally stabilized EGF and bFGF on wound healing in type I and type II diabetic mice. Acta Biomater 2018; 66: 325-34.

29. Lv Ch, Wu J, He H. Experimental study on the expression of IL-1 $\beta$ and bFGF in wound healing process of rabbit cutaneous infective wound in Liu-He-Dan. Evid Based Complement Alternat Med 2017; 2017: 7230178.

30. Gazaerly HE, Elbardisey DM, Eltokhy HM, Teaama D. Effect of transforming growth factor beta 1 on wound healing in induced diabetic rats. Int J Health Sci 2013; 7: 160-72.

31. Wang L, Qin W, Zhou Y, et al. Transforming growth factor $\beta$ plays an important role in enhancing wound healing by topical application of povidone-iodine. Sci Rep 2017; 7: 991.
32. Lal BK, Saito S, Pappas PJ, et al. Altered proliferative responses of dermal fibroblasts to TGF- $\beta 1$ may contribute to chronic venous stasis ulcer. J Vasc Surg 2003; 37: 1285-93.

33. Santoro MM, Gaudino G. Cellular and molecular facets of keratinocyte reepithelization during wound healing. Exp Cell Res 2005; 304: 274-86.

34. Peplow PV, Chatterjee MP. A review of the influence of growth factors and cytokines in in vitro human keratinocyte migration. Cytokine 2013; 62: 1-21.

35. Uchi H, Igarashi A, Urabe K, et al. Clinical efficiency of basic fibroblast growth factor (bFGF) for diabetic ulcer. Eur J Dermatol 2009; 19: 461-8.

36. Jude EB, Blakytny R, Bulmer J, et al. Transforming growth factor - beta 1, 2, 3 and receptor type I and II in diabetic foot ulcers. Diabet Med 2002; 19: 440-7.

37. Tuttolomondo A, La Placa S, Di Raimondo D, et al. Adiponectin, resistin and IL-6 plasma levels in subjects with diabetic foot and possible correlations with clinical variables and cardiovascular co-morbidity. Cardiovasc Diabetol 2010; 9: 1-7.

38. Xiao Y, Zheng X, Zou X, et al. Extracellular vesicles in type 2 diabetes mellitus key roles in pathogenesis, complications, and therapy. J Extracell Vesicles 2019; 8: 1625677.

39. Stomka A, Urban SK, Lukacs-Kornek V, et al. Large extracellular vesicles: have we found the holy grail of inflammation? Front Immunol 2018; 9: 2723

40. Yang D, Dias D, Dias P. Exosomes or microvesicles, a secreted subcellular organelle contributing to inflammation and diabetes. Diabetes 2018; 67: 2154-6. 